\title{
Gastric Cancer Case with Skin Metastases and Scleroderma-like Paraneoplastic Rheumatic Syndrome
}

\author{
Alise Lavrinovica, Evita Gasenko, and Signe Plate
}

\section{ABSTRACT}

In this case report, we will describe a gastric cancer case, which presented with rare manifestation of skin metastases and unrelated to the mechanical impact of distant metastases scleroderma-like paraneoplastic rheumatic syndrome.

72-year-old woman was referred to hospital with complaints gradual during the time period of one year. Skin changes included - thickening, dermal induration mostly seen on her upper body - arms, neck, face, chest and abdominal wall, causing contracture and limited movements. In the area of her upper arms, neck and upper part of her chest multiple small, painless, hard subcutaneous nodules were present and hyperpigmentation in the neck area. There were no signs of Raynaud's phenomenon, telangiectasia, alopecia, hypopigmentation or muscle weakness and pain. Upon further examination and multiple biopsies (gastric and skin nodules) poorly differentiated gastric carcinoma was confirmed with peritoneal dissemination, ascites, cutaneous, subcutaneous metastasis and scleroderma-like paraneoplastic syndrome. After systemic treatment for gastric cancer patient showed improvement of paraneoplastic syndrome manifestations, quality of life and radiologically stable disease.

Keywords: gastric cancer, paraneoplastic syndrome, scleroderma, cutaneous metastasis.

\section{INTRODUCTION}

Gastric cancer can spread locally to adjuvant structures and also develop distant metastatic deposits mainly by hematogenous spread. It usually metastasizes to the liver, peritoneal cavity, and regional lymph nodes more often than to the skin [1]. The incidence of cutaneous metastasis from carcinomas of the upper digestive tract has been reported to be less than 1\%. Umbilical metastasis, Sister Mary Joseph's nodule, is the typical site for a cutaneous tumor resulting from metastasis of gastric cancer. Apart from the umbilical metastasis, the most common clinical presentation of gastric carcinoma metastatic to the skin is as multiple discrete, slow-growing, hard nodules [2].

One of the rare findings in malignant neoplasms are paraneoplastic rheumatological syndromes. Term paraneoplastic syndrome refers to symptoms caused by a malignancy but which are not directly related to the tumor mass nor its metastasis [3]. The association between malignancy and rheumatic diseases is complex. A two-way relationship exists: 1) malignancy can arise in the setting of preexisting rheumatic disease or as a result of immunosuppressive drugs used for the treatment of rheumatic diseases; and 2) rheumatic diseases due to metastasis to musculoskeletal system or nonmetastatic paraneoplastic rheumatic diseases or rheumatic diseases which developed as complications from malignancy treatments [3], [4].

Paraneoplastic syndromes are the expression of an underlying cancer, frequently occult, and can be caused by a wide variety of remote tumor effects unrelated to the mechanical impact of the tumor mass or distant metastases. They result from substances released from tumor cells like hormones, peptides, antibodies, or from immunologic and other host reactions to the tumor [4]-[7].

In this case report, we will describe a gastric cancer case, which presented with skin metastases and unrelated to the mechanical impact of distant metastases scleroderma-like paraneoplastic rheumatic syndrome.

\section{CASE REPORT}

A 72-years-old, Caucasian, female was admitted to the regional hospital (February 2019) with complaints of gradual weight loss of $30 \mathrm{~kg}$ in total during the last year, decreased appetite and skin changes. Her underlying comorbidities were type 2 diabetes mellitus (satisfactory control was achieved with gliclazide MR $60 \mathrm{mg}$ once daily and vildagliptin $50 \mathrm{mg} / \mathrm{metformin}$ hydrochloride $1000 \mathrm{mg}$ twice daily) and arterial hypertension grade 2 (treated with 
perindopril $20 \mathrm{mg}$ once daily and nebivolol $5 \mathrm{mg}$ once daily). Patient was never smoker, was drinking very rarely in moderation and had no prior personal or family history of cancer. Initial blood count showed grade I normochromic normocytic anaemia, abdominal ultrasound exam revealed chronic calculous cholecystitis, upper endoscopy showed ulceration in gastric body (histological report stated insufficient tissue for assessment). Laparoscopic cholecystectomy was performed. During procedure it was described that gallbladder was fixed in numerous adhesions, histological report was not available.

Patient's skin changes had developed gradually over the time course of around one year (since March 2018). Skin thickening, dermal induration was mostly seen on her upper body - arms, neck, face, chest and abdominal wall skin. Radial furrowing around the mouth was present, which limited the aperture of the mouth (microstomy). Eyelid movement was not limited. Mild hyperpigmentation was observed in the neck area (Fig. 1).

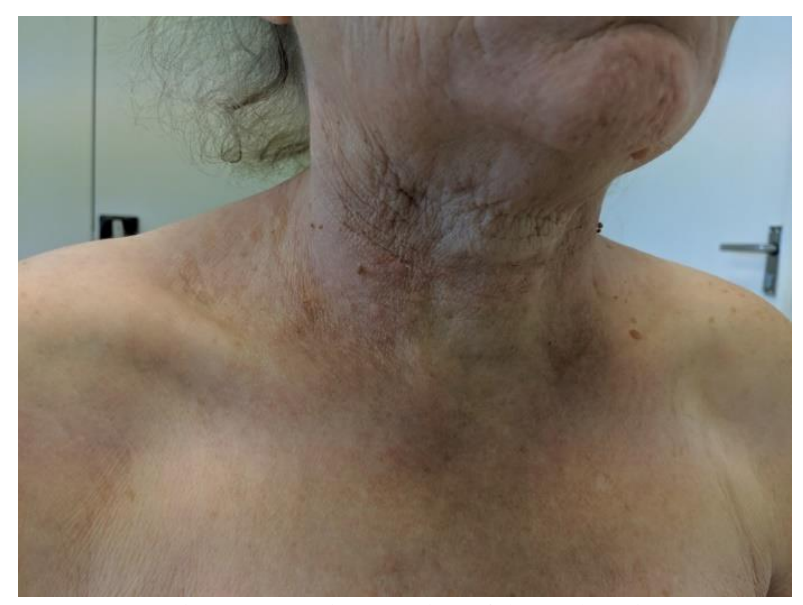

Fig. 1. Hyperpigmentation of the neck region.

Skin thickening in the arms increased proximally patient's fingers were not swollen and there were no significant skin changes until the antecubital fossa area where thickening and induration was causing contracture and limited movements (Fig. 2).

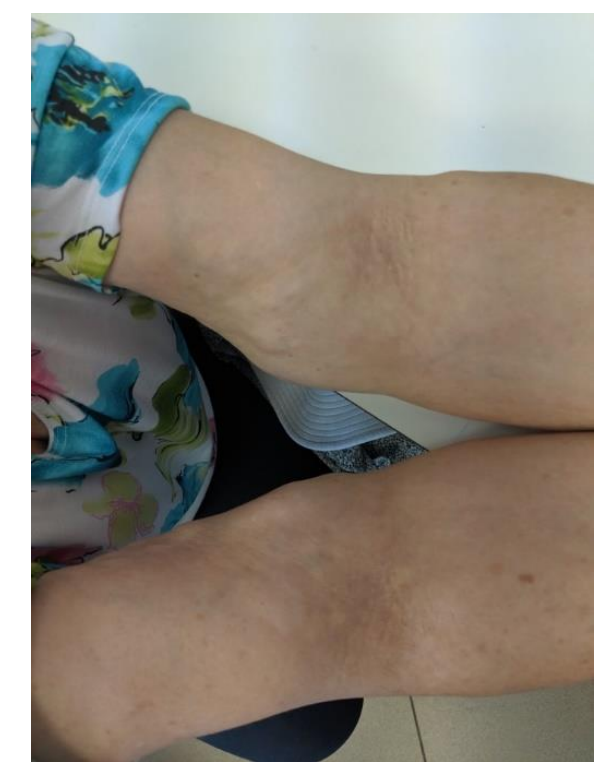

Fig. 2. Thickening of the skin and contractures of the antecubital fossa.
In the area of her upper arms, neck and upper part of her chest skin had thickened and multiple small, painless, hard subcutaneous nodules were present. Due to substantial induration of the skin in neck area patient had very limited head motions. Similarly, induration without palpable nodules was seen in the upper part of her abdominal wall (Fig. 3).

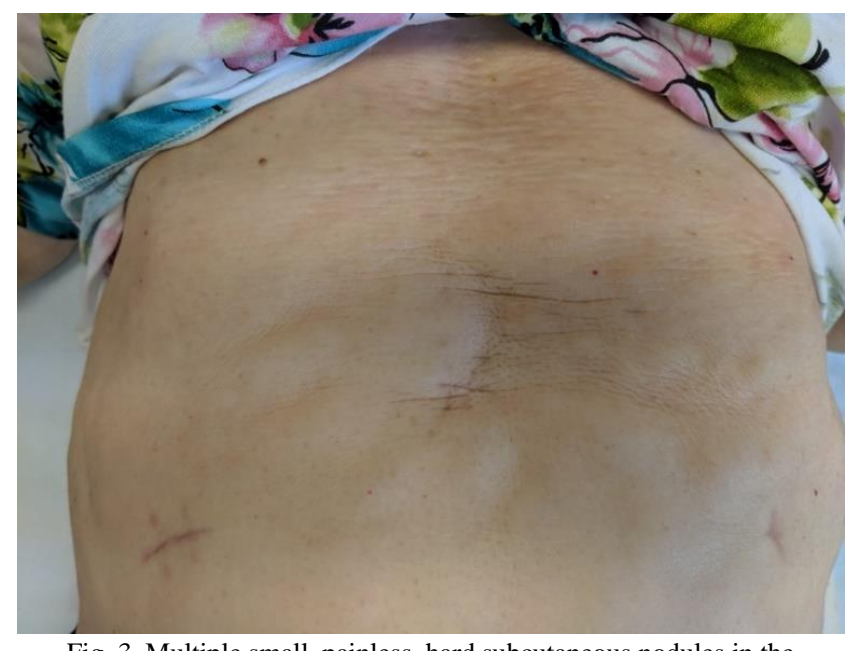

Fig. 3. Multiple small, painless, hard subcutaneous nodules in the abdominal wall.

Her skin was dry, but no dryness of conjunctiva or oral mucosa was observed. Skin in the lower part of her body was thin and dry but without substantial induration areas. There were no signs of Raynaud's phenomenon, telangiectasia, alopecia, hypopigmentation or muscle weakness and pain.

After cholecystectomy patient was referred to the tertiary care hospital for further evaluation (March 2019) as connective tissue disease - systemic sclerosis was suspected. Skin biopsy of the chest wall was performed. It showed no signs of connective tissue disease but presence of carcinoma cells (positive for CK1/3AE, CK7 and negative for CK20, CD68) and fibrosis with suspected primary localisation of the tumour in breast, oesophagus, stomach, pancreas, biliary and urogenital tract.

This finding prompted further investigations: barium $\mathrm{x}-$ rays of the gastrointestinal tract suggested scirrhous gastric malignancy. For the second time upper endoscopy was performed showing ulceration in gastric body, antrum, lesser curvature, biopsy was taken (histology showed no malignant cells). Abdominal MRI scan showed thickened wall of stomach, colon ascendens, colon transversum, peritoneal dissemination and ascites. Chest CT showed no abnormalities. Patient was assigned to diagnostic laparoscopy and biopsy. Pathohistological analysis showed poorly differentiated (grade 3) carcinoma metastasis in omentum and lymph node. Third upper endoscopy was performed with multiple biopsies, this time confirming poorly differentiated carcinoma (grade 3). Her-2 testing was not performed. Final clinical diagnosis was gastric cancer $\left(\mathrm{T}_{4} \mathrm{~N}_{1} \mathrm{M}_{1}\right)$ stage IV, peritoneal dissemination, ascites, cutaneous, subcutaneous metastasis and scleroderma-like paraneoplastic syndrome. Patient was prescribed prednisolone $20 \mathrm{mg}$ once daily. 
Multidisciplinary tumour board offered palliative chemotherapy. Upon evaluation by medical oncologist patient's performance status was ECOG 1, weight $67 \mathrm{~kg}$, height $158 \mathrm{~cm}$ (BSA $1.6 \mathrm{~m}^{2}$ ), blood count showed normochromic normocytic anemia grade 1 , thrombocytosis (platelet count $526 \times 10^{9} / \mathrm{L}$ ), increased alkaline phosphotase grade 2, increased ALAT and ASAT grade 1, hyperglycemia grade $2(7,5 \mathrm{mmol} / \mathrm{L})$, CEA - 31,4 ng/mL, CA $19.9<2,5 \mathrm{U} / \mathrm{mL}$. Patient received chemotherapy Day 1: epirubicin $50 \mathrm{mg} / \mathrm{m}^{2} \mathrm{i} / \mathrm{v}$, oxaliplatin $130 \mathrm{mg} / \mathrm{m}^{2} \mathrm{i} / \mathrm{v}$ and D13: 5 -FU $800 \mathrm{mg} / \mathrm{m}^{2}, 21 \mathrm{~d}$ cycle. After $2^{\text {nd }}$ cycle of chemotherapy clinical improvement was observed patient's skin became more elastic, she could open her mouth fully and mobility in arms and neck increased, subcutaneous nodules were less prominent and she slowly started to gain weight (up to $76 \mathrm{~kg}$, then it remained stable). After four cycles of chemotherapy CT of thorax, abdomen and pelvis was performed and according to RECIST 1.1 criteria showed stable disease (decreased thickness of the gastric wall, peritoneal dissemination and ascites). As patient's quality of life and CT scans improved, she received additional four cycles of chemotherapy. Patient did not report any chemotherapy side effects besides alopecia, laboratory tests showed periodic increase in ALAT and ASAT up to grade 2 and EKG upon two occasions showed supraventricular extrasystoles (asymptomatic). Second CT scan also showed stable disease. Patient completed eight cycles of chemotherapy from June 2019 until December 2019 and in January continued therapy with 1-(2tetrahydrofuryl)-5-fluorouracil $400 \mathrm{mg}$ (Tegafur) oral capsules. Control visit and CT scan is planned in April 2020. Unfortunately, patient died in April 2020. Patient's overall survival was 14 months.

\section{DISCUSSION}

Our patient had undergone three endoscopic biopsies. Only one out of three biopsies have shown cancer cells. The possible reason why two biopsies has not recognized cancer could be too deep localization of tumor, so therefore during endoscopic procedure it was not possible to reach tumor tissues. There is an alternative option to use endoscopic ultrasound combined with a fine-needle aspiration in this type of cases.

Overall, dysplasia appeared to be more easily detected with significance in comparison to early gastric cancer or advanced gastric cancer, and with cumulative yield analysis, advanced gastric cancer appeared to have the highest falsenegative rate. These findings are consistent to previous reports, in which earlier-stage lesions were detected more readily [18].

Stage IV gastric cancer has a poor prognosis with oneyear survival rate $40 \%$, two years $-17 \%$, three years $-10 \%$, four years $-8 \%$ and five years $-7 \%$ (according to the AJCC Cancer Staging Manual 6th Edition) Cutaneous metastasis is still a poor prognostic sign. The general survival after the diagnosis of skin metastasis drops below one year (average 11.4 weeks with a range of 2 to 34 weeks) and deteriorates further with multiple cutaneous metastases [8]. Patient described in our clinical case reached one-year survival bar and exceeded it.
It is estimated that paraneoplastic syndromes affect up to $8 \%$ of patients with cancer [9], [10]. The incidence of paraneoplastic syndrome is much less than $1 \%$ for solid tumors, except for small-cell lung cancer and thymoma [11]. The paraneoplastic syndromes are classified into endocrine, neurologic, dermatologic, rheumatologic, and hematologic syndromes [12]. In malignant digestive neoplasms paraneoplastic dermatologic syndromes are the most common [13]. In our case patient suffered from the rheumatological paraneoplastic syndrome rare for gastrointestinal tumors.

In the available literature there are numerous reports on the coexistence of neoplasm and paraneoplastic rheumatic syndromes including dermatomyositis and polymyositis, polymyalgia rheumatica, eosinophilic fasciitis, erythema nodosum, Sjögren's syndrome with monoclonal gammopathy, steroid-resistant leukocytoclastic vasculitis, asymmetric arthritis in elderly patients, arthritis with monoclonal gammopathy, sacroiliitis, Still's disease in adults, antiphospholipid syndrome, mixed cryoglobulinaemia, Raynaud's phenomenon in patients aged 50 years or more, or systemic sclerosis [14].

Skin lesions similar to those present in systemic sclerosis have been found in patients with malignant tumors and other diseases, defined by the terms pseudoscleroderma or pseudosclerosis. Lung, breast and stomach cancer, plasmacytoma and carcinoids can be associated with this paraneoplastic syndrome. In several cases it is necessary to differentiate systemic sclerosis from a paraneoplastic syndrome; the latter is more probable in the case of history of cancer, exposure to carcinogens, onset of symptoms in elderly people, systemic symptoms (fever, fatigue, weight loss). Therefore, paraneoplastic syndromes should be suspected in elderly patients, whose clinical symptoms, laboratory/instrumental findings, or therapeutic response diverge from what is usually expected [15]. In the case of occurrence of systemic scleroderma in elderly patients, two possibilities shall be considered: paraneoplastic syndrome or idiopathic scleroderma (in the course of which cancer is very likely to be diagnosed in the following years) [16]. Our 72-year-old patient suffered from weight loss, decreased appetite and skin changes and she was highly suspicion of neoplasm.

In pseudoscleroderma, histopathology is not discerning from real scleroderma. There are no capillaroscopic abnormalities or distinctive antibodies from scleroderma. Raynaud's phenomenon and oesophageal involvement are rare. Diagnostic features are considered failure to respond to standard treatment and remission of symptoms after cancer treatment, coupled with their reappearance when the neoplasms recur [17].

\section{CONCLUSIONS}

Early recognition of paraneoplastic syndrome is crucial to find out oncological disease and start treatment. That improves the patient's life duration and quality. Our patient showed improvement of her paraneoplastic syndrome and stable oncological disease despite advanced disease and poor prognosis. Besides our patient exceeded the median survival rate. She survived 14 months from the moment of 
diagnosis. It is also very essential to take biopsy correctly to avoid false results, because it can delay diagnosis. stratified by biopsy number and site. Med (United States). 2016;95(30):e4196. doi:10.1097/MD.0000000000004196.

\section{DECLARATIONS}

All procedures performed in studies involving human participants were in accordance with the ethical standards of the institutional and/or national research committee and with the 1964 Helsinki declaration and its later amendments or comparable ethical standards.

The authors declare that they have no competing interests.

Funding: none.

All authors have contributed significantly and are in agreement with the content of the manuscript.

\section{REFERENCES}

[1] Esaki Y, Hirayama R, Hirokawa K. A comparison of patterns of metastasis in gastric cancer by histologic type and age. Cancer. 1990;65(9):2086-2090. doi:10.1002/10970142(19900501)65:9<2086::AID-CNCR2820650933>3.0.CO;2-B.

[2] Sugathan P, Martin A. Mudi-Chood: On the forearm. Indian $J$ Dermatol. 2011;56(2):228-229. doi:10.4103/0019-5154.80437.

[3] Omer FŞ. Paraneoplastic Rheumatic disorders. Turkish J Rheumatol. 2012;27(1):18-23. doi:10.5606/tjr.2012.002.

[4] Azar L, Khasnis A. Paraneoplastic rheumatologic syndromes. Curr $\begin{array}{lll}\text { Opin Rheumatol. } & \text { 2013;25(1):44-49. }\end{array}$ doi:10.1097/BOR.0b013e328359e780.

[5] Szekanecz Z, Szekanecz É, Bakó G, Shoenfeld Y. Fax +416130612 34 E-Mail karger@karger.ch Clinical Section / Mini-Review Malignancies in Autoimmune Rheumatic Diseases-A Mini-Review. Published online 2010. doi:10.1159/000314634.

[6] Wen J, Ouyang H, Yang R, et al. Malignancy dominated with rheumatic manifestations: A retrospective single-center analysis. $S c i$ Rep. 2018;8(1):1786. doi:10.1038/s41598-018-20167-w.

[7] Racanelli V, Prete M, Minoia C, Favoino E, Perosa F. Rheumatic disorders as paraneoplastic syndromes is. Published online 2008. doi:10.1016/j.autrev.2008.02.001.

[8] Cutaneous metastasis of gastric adenocarcinoma: An exuberant and unusual clinical presentation. Accessed October 24, 2020. https://escholarship.org/uc/item/1xs1g51p.

[9] Pelosof LC, Gerber DE. Paraneoplastic syndromes: An approach to diagnosis and treatment. Mayo Clin Proc. 2010;85(9):838-854. doi:10.4065/mcp.2010.0099.

[10] Hakkou J, Rostom S, Bahiri R, Hajjaj-Hassouni N. Paraneoplastic rheumatic syndromes: Report of eight cases and review of literature. Rheumatol Int. 2012;32(6):1485-1489. doi:10.1007/s00296-0112252-9.

[11] Murakami H, Rino Y, Yamanaka S, et al. Paraneoplastic neurological syndrome in a patient with gastric cancer. Gastric Cancer. 2010;13(3):204-208. doi:10.1007/s10120-010-0563-2.

[12] Baijens LWJ, Manni JJ. Paraneoplastic syndromes in patients with primary malignancies of the head and neck. Four cases and a review of the literature. Eur Arch Oto-Rhino-Laryngology. 2006;263(1):3236. doi:10.1007/s00405-005-0942-1.

[13] Adams DH. Sleisenger and Fordtran's Gastrointestinal and Liver Disease. Gut. 2007;56(8):1175-1175. doi:10.1136/gut.2007.121533.

[14] Ciołkiewicz M, Domysławska I, Ciołkiewicz A, Klimiuk PA, Kuryliszyn-Moskal A. Coexistence of systemic sclerosis, scleroderma-like syndromes and neoplastic diseases. Pol Arch Med Wewn. 2008;118(3):119-126. doi:10.20452/pamw.330.

[15] Manzini CU, Colaci M, Ferri C, Manzini E. Paraneoplastic rheumatic disorders: A narrative review. Reumatismo. 2018;70(4):199-211. doi:10.4081/reumatismo.2018.1069.

[16] Marek M, Rudny R. Scleroderma of geriatric age and sclerodermalike paraneoplastic syndrome - Description of two cases. Reumatologia. 2016;54(2):91-94. doi:10.5114/reum.2016.60220.

[17] Ferreli C, Gasparini G, Parodi A, Cozzani E, Rongioletti F, Atzori L. Cutaneous Manifestations of Scleroderma and Scleroderma-Like Disorders: a Comprehensive Review. Clin Rev Allergy Immunol. 2017;53(3):306-336. doi:10.1007/s12016-017-8625-4.

[18] Kwack WG, Ho WJ, Kim JH, et al. Understanding the diagnostic yield of current endoscopic biopsy for gastric neoplasm: A prospective single-center analysis based on tumor characteristics 